\title{
The platelet-lymphocyte ratio is a promising predictor of early postoperative acute kidney injury following cardiac surgery: a case-control study
}

\author{
Wenyu $\mathrm{He}^{1,2 \wedge}$, Yingling Zhou ${ }^{1,2}$ \\ ${ }^{1}$ School of Medicine, South China University of Technology, Guangzhou, China; ${ }^{2}$ Guangdong Provincial People's Hospital, Guangdong Academy of \\ Medical Sciences, Guangzhou, China \\ Contributions: (I) Conception and design: W He; (II) Administrative support: Y Zhou; (III) Provision of study materials or patients: W He; (IV) \\ Collection and assembly of data: W He; (V) Data analysis and interpretation: W He; (VI) Manuscript writing: Both authors; (VII) Final approval of \\ manuscript: Both authors. \\ Correspondence to: Dr. Yingling Zhou. Guangdong Provincial People’s Hospital, Guangdong Academy of Medical Sciences, Guangzhou 510080, \\ China; School of Medicine, South China University of Technology, Guangzhou 510006, China. Email: zylgdh@163.com.
}

Background: Acute kidney injury (AKI) is a common complication following cardiac surgery. This study explored the correlation between hematological biomarkers and the occurrence of postoperative AKI following cardiac surgery.

Methods: This was a retrospective case-control, single-center study. This study enrolled 91 patients who underwent cardiac surgery with cardiopulmonary bypass (CPB) support in Guangdong Provincial People's Hospital Zhuhai Hospital between March 2019 and July 2021. The baseline serum creatinine levels of the patients was $<132.6 \mu \mathrm{mol} / \mathrm{L}$. The patients' electronic medical records were retrospectively reviewed. AKI was diagnosed according to the Kidney Disease Improving Global Outcomes (KDIGO) 2012 Acute Kidney Injury Guideline criteria. Patients who sustained AKI in the 48 hours following cardiac surgery were categorized as Group A ( $\mathrm{n}=48)$, while patients with normal serum creatinine levels postoperatively were categorized as Group B ( $n=43)$. The baseline demographic and clinical characteristics of the two groups were compared. Univariate analysis was performed to evaluate the correlation between biomarkers and postoperative AKI. Multivariate logistic regression was performed to identify the predictors of AKI following cardiac surgery.

Results: Forty-eight patients were diagnosed with AKI during the first 48 hours after cardiac surgery, while 43 patients were found to have normal creatinine levels postoperatively. Multivariate logistic regression analysis revealed advanced age $(\mathrm{P}=0.007)$, preoperative increased creatinine $(\mathrm{P}=0.023)$, duration of intraoperative hypotension $(\mathrm{P}=0.027)$, and the platelet-lymphocyte ratio ( $\mathrm{P} / \mathrm{LR} ; \mathrm{P}=0.042)$ as predictors of AKI following cardiac surgery with CPB. This study was performed in a single center, which might not be generalized to whole population. Ongoing review and modification of the surgical protocols may contribute to bias of the study.

Conclusions: The P/LR can be obtained from a routine blood test, and may potentially be utilized as an independent indicator of AKI following cardiac surgery.

Keywords: Cardiac surgery; acute kidney injury (AKI); cardiopulmonary bypass (CPB); platelet-lymphocyte ratio (P/LR); neutrophil-lymphocyte ratio (N/LR)

Submitted Aug 19, 2021. Accepted for publication Dec 08, 2021.

doi: $10.21037 / \mathrm{atm}-21-6012$

View this article at: https://dx.doi.org/10.21037/atm-21-6012

^ ORCID: 0000-0003-0351-267X. 


\section{Introduction}

Cardiac surgery-associated acute kidney injury (CSA-AKI) is not an uncommon complication following cardiac surgery. $\mathrm{AKI}$ is also an independent risk factor of other postoperative adverse events, resulting in high fatality rate (1). The incidence of AKI following cardiac surgery was previously stated at $5-42 \%$ (2), with approximately $1-2 \%$ of these patients requiring renal replacement therapy (3). AKI is a complex syndrome that varies from minimally deranged serum creatinine to anuresis. In order to identify patients at risk of AKI, studies have been conducted to explore biomarkers that are indicative of CSA-AKI. Multiple factors have been implicated, including advanced age, chronic kidney disease, aortic cross-clamping time, duration of cardiopulmonary bypass (CPB) time, intraoperative blood pressure, and blood transfusion (4-6).

The exact pathogenesis of CSA-AKI is not fully understood; however, systemic inflammatory response was considered to play an important role in the development of CSA-AKI (7). Platelet activation is currently found pivotal in the process of inflammatory response by adhering to endothelium and recruiting neutrophils. A fall in platelet counts and rise in neutrophils are commonly observed in patients underwent cardiac surgery with CPB support. The nadir of early postoperative platelet count was also reported to be associated with increased incidence of AKI (8). The neutrophil-lymphocyte ratio $(\mathrm{N} / \mathrm{LR})$ and platelet-lymphocyte ratio (P/LR) are low-cost systemic inflammatory markers that are easily calculated from a complete blood count. Recently, some correlations between elevated N/LR or P/LR and the outcomes of some critical illnesses (such as septic shock) have been reported (9). Deranged N/LR levels have also been described in patients with AKI following major abdominal surgery (10).

In terms of the distinct pathophysiological changes in patients who undergo cardiac surgery, the correlation between CSA-AKI and these blood cell count ratios has yet to be explored. Hence, this study aims to determine the connection between the development of CSAAKI and the N/LR and P/LR values. We also explored the potential utility of N/LR and P/LR for predicting postoperative AKI in the background of cardiac surgery. We present the following article in accordance with the STROBE reporting checklist (available at https://dx.doi. org/10.21037/atm-21-6012).

\section{Methods}

\section{Study design and setting}

This study was designed as a single-center retrospective case-control study, and was conducted at Guangdong Provincial Hospital Zhuhai Hospital. The medical records of patients who underwent cardiac surgery between March 2019 and April 2021 were retrospectively reviewed. The operations were performed by the cardiothoracic surgical team of Guangdong Provincial Hospital Zhuhai Hospital. After surgery, all patients were transferred to the intensive care unit (ICU) for further monitoring. As per local surgical routine, a full blood count and biochemical study [which contains creatinine, procalcitonin (PCT), brain natriuretic peptide (BNP), and troponin I (CTnI) levels] were measured at four separate time points during the perioperative period, including the preoperative period, as well as at $0-6,6-12$, and $12-24$ hours postoperatively. There were no additional interventions from the investigators. According to local policy, informed consent was waived due to the retrospective nature of the study. All the procedures performed in this study were in accordance with the Declaration of Helsinki (as revised in 2013). The study was approved by institutional review board of School of Medicine, South China University of Technology.

\section{Participants}

Patients aged between 18 and 80 years with a preoperative baseline serum creatinine level $>132.6 \mu \mathrm{mol} / \mathrm{L}$ were included. All patients enrolled in this study were admitted to the ICU postoperatively and remained there for 48 hours. Patients diagnosed with chronic kidney disease, congestive heart failure (left ventricular ejection fraction less than $30 \%$ ), cerebrovascular disease in the last 30 days, chronic obstructive pulmonary disease, acute infections, malignancy, thyroid disorders, systemic inflammatory disease, and preoperative hematological disorder were excluded. Patients who underwent beating heart cardiac surgery were also excluded.

\section{Diagnosis}

According to diagnostic criteria of the Kidney Disease Improving Global Outcomes (KDIGO), AKI can be established by comparing the postoperative serum creatinine levels with the preoperative serum creatinine levels. If the 
postoperative serum creatinine increased by more than $26.5 \mu \mathrm{mol} / \mathrm{L}$ within 48 hours following the cardiac surgery, or the postoperative serum creatinine increased to more than 1.5 times the preoperative creatinine level, a diagnosis of CSA-AKI can be made. The peak serum creatinine level during the first 2 days following surgery was employed for the diagnosis of AKI. Patients who were diagnosed with AKI were categorized as Group A, while patients who did not develop AKI were categorized as Group B.

Hypertension was considered in cases with blood pressure $>140 / 90 \mathrm{mmHg}$ or those involving use of antihypertensive medications. Diabetes was considered if fasting blood glucose was $>126 \mathrm{mg} / \mathrm{dL}$ or in cases involving the use of anti-diabetics. Hyperlipidemia was considered if the total cholesterol level was $>220 \mathrm{mg} / \mathrm{dL}$ and low-density lipoprotein (LDL)-cholesterol $>130 \mathrm{mg} / \mathrm{dL}$, or in cases involving the use of anti-hyperlipidemic medications.

\section{Laboratory parameters}

Hematologic parameters were obtained by collecting $4 \mathrm{~mL}$ venous blood samples with an ethylene diamine tetraacetic acid (EDTA) tube, and the cellular counts were calculated using an automated blood count device (Mindray, Model: BC-5390 CRT ST 67000698, China) in the hematology lab. P/LR was acquired by dividing the platelet count by the lymphocyte counts, and N/LR was obtained by dividing the neutrophil count by the lymphocyte count.

\section{Operative techniques}

Intraoperatively, all patients enrolled in our study were under general anesthesia and $\mathrm{CPB}$ with aortic and venous cannulations. Activated clotting time (ACT) was monitored during the operation. Standard CPB circuits were used. Antegrade hypothermic and hyperkalemic blood cardioplegia were used. CPB flow was kept between 2.2 and $2.5 \mathrm{~L} / \mathrm{min} / \mathrm{m}^{2}$, and hematocrit was kept between 0.25 and 0.3 during CPB. The types of operation included valvular replacement, valvular plastic, and coronary artery bypass.

\section{Statistical analysis}

SPSS software version 22.0 (SPSS Inc., Chicago, IL, USA) was used for statistical analysis. Measurement and count data were described as the median and range from minimum to maximum. Data acquired by calculation were described as percentages (\%). Measurement data that was normally distributed was assessed using the KolmogorovSmirnov test, while Levene's test was employed to assess the homogeneity of distribution for equality of variance. The Student's $t$-test was used to evaluate the differences between groups that were normally distributed with homogeneity. The Mann-Whitney U test was used to evaluate differences between groups that were non-normally distributed or homogeneous if applicable. Parametric or non-parametric Pearson chi-square test or Fisher's exact test were used to assess difference between groups in count data. The effects of the risk factors that were suggested to be influential were investigated using univariate regression analyses. From the results, the multiple effects of the risk factors that were suggested to be influential in predicting the CSA-AKI were studied using retrospective selective multivariate logistic regression analysis. The multivariate logistic regression analysis also indicated the odds ratio (OR), 95\% confidence interval (CI), and the significant level for each risk factor. The sensitivity and the specificity of P/LR and N/LR in predicting early-stage AKI was demonstrated using a receiver operating characteristic (ROC) curve. Results were defined as statistically significant when $\mathrm{P}<0.05$.

The number of cases during 2019 and 2021 determined the sample size. To handle missing data, a mean substitution was employed. The mean value of a variable was used to replace the missing data value for the same variable. Detection bias could lead to confounding between AKI and risk factors. To address bias, results of blood tests were recorded at certain period of time instead of being collected at fixed specific time.

\section{Results}

Table 1 depicts the preoperative demographic and clinical data of the patients. No differences were observed between the two groups in terms of their preoperative clinical conditions. However, the age of Group A was more advanced compared to Group B ( $\mathrm{P}=0.007)$.

According to the KDIGO criteria, 48 patients were diagnosed with CSA-AKI. Forty-four (91.6\%) patients were classified as Stage-I, $3(6.3 \%)$ as Stage-II, and 1 (2\%) as Stage-III. Table 2 summarizes the preoperative hematological parameters of the two groups. Significant differences were observed in the creatinine levels $(\mathrm{P}=0.023)$ and urea levels $(\mathrm{P}=0.017)$ between the two groups.

The patients' postoperative blood test results are shown in Table 3. The N/LR between 0-6 hours postoperatively $(\mathrm{P}=0.034), \mathrm{P} / \mathrm{LR}$ at $6-12$ and $12-24$ hours postoperatively 
Table 1 Patients' demographic and clinical data



*, Pearson chi-square test or Fisher's exact test; ${ }^{\star \star}$, Student's $t$-test. AKI, acute kidney injury; BMI, body mass index; CVA, cerebral vascular accident.

Table 2 Preoperative laboratory results

\begin{tabular}{|c|c|c|c|}
\hline Preoperative laboratory results & Group A: AKI $(n=48)$, median [min-max] & Group B: non-AKI $(n=43)$, median [min-max] & $P$ value ${ }^{\star \star}$ \\
\hline Preoperative hematocrit (\%) & $41.8[32.5-53.4]$ & 40.7 [29.2-47.3] & 0.240 \\
\hline Preoperative creatinine ( $\mu \mathrm{mol} / \mathrm{L})$ & $90.4[47-155]$ & $74.7[36-133]$ & 0.001 \\
\hline Preoperative urea (mmol/L) & $6.7[3.7-14.1]$ & $5.6[3.2-13.1]$ & 0.017 \\
\hline Preoperative platelet count $\left(\times 10^{9} / \mathrm{L}\right)$ & $194.3[101-331]$ & 212.4 [96-352] & 0.131 \\
\hline Preoperative CRP (mg/L) & $10.3[0-168]$ & $3.5[0-25.15]$ & 0.097 \\
\hline Preoperative lymphocyte count $\left(\times 10^{9} / L\right)$ & $1.6[0.67-3.51]$ & $1.7[0.68-3.1]$ & 0.422 \\
\hline Preoperative N/LR & $3.5[0.82-20.0]$ & $2.5[0.34-7.838]$ & 0.117 \\
\hline
\end{tabular}

${ }^{* *}$, Student's $t$-test. AKI, acute kidney injury; CRP, C-reactive protein; N/LR, neutrophil-lymphocyte ratio; P/LR, platelet-lymphocyte ratio.

( $\mathrm{P}=0.017$ and 0.035 , respectively), change in $\mathrm{N} / \mathrm{LR}$ at $0-6$ hours postoperatively $(\mathrm{P}=0.016)$, and change in $\mathrm{P} / \mathrm{LR}$ at $0-6$ and $6-12$ hours postoperatively $(\mathrm{P}=0.011$ and 0.027 , respectively) were significantly different between the two groups.

In terms of the biochemical parameters, BNP levels at 6, 12 , and 24 hours postoperatively $(\mathrm{P}=0.013,0.014$ and 0.002 respectively), PCT levels at 6 and 12 hours postoperatively ( $\mathrm{P}=0.012$ and 0.017 respectively), and CTnI level at 24 hours postoperatively $(\mathrm{P}=0.002)$ differed significantly between the two groups. Due to high missing rate of PCT between 0 and 6 hours postoperatively, this variable was removed from database.

Table 4 illustrates the intraoperative data of the two 
Table 3 Postoperative blood test of patients

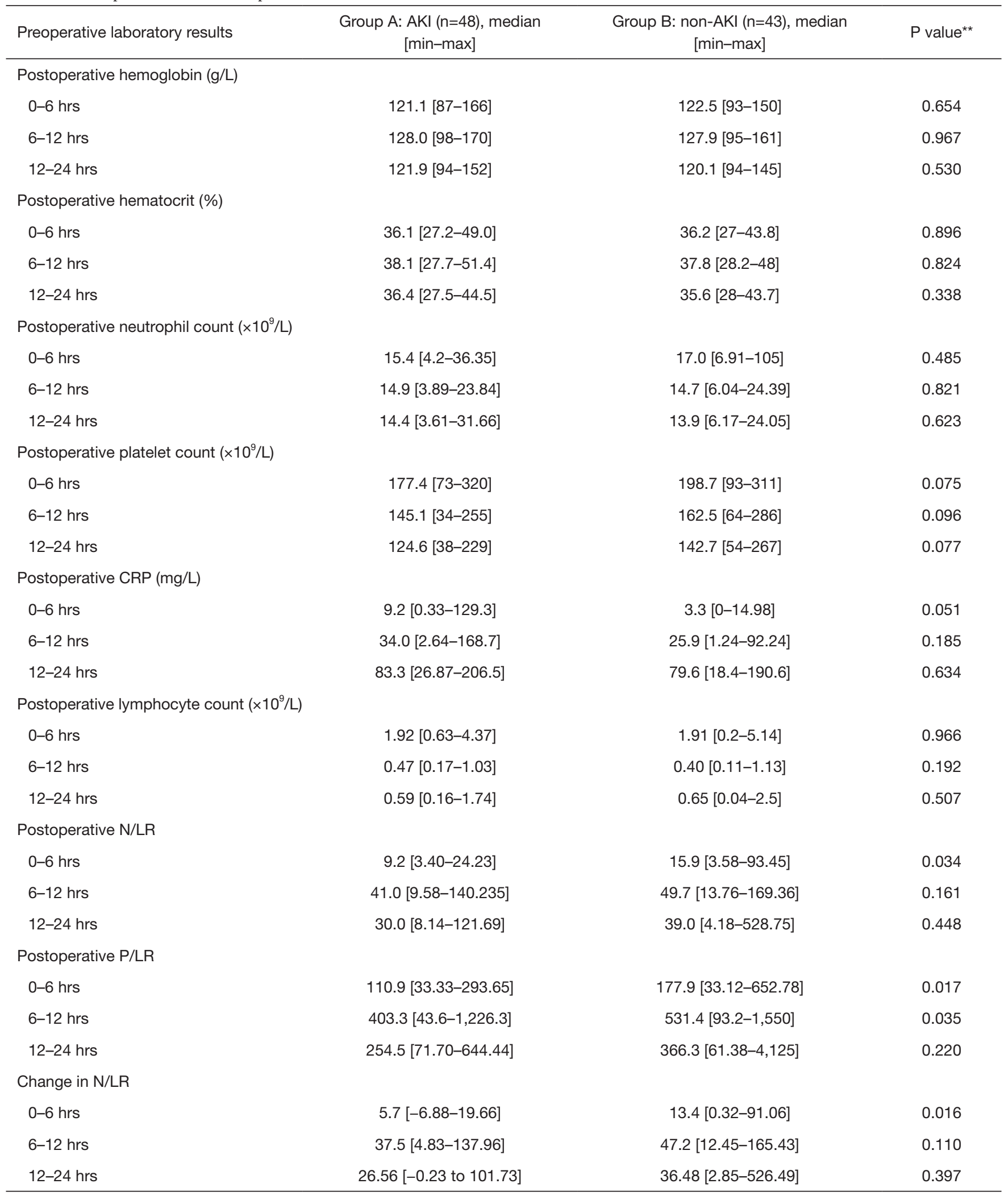

Table 3 (continued) 
Table 3 (continued)

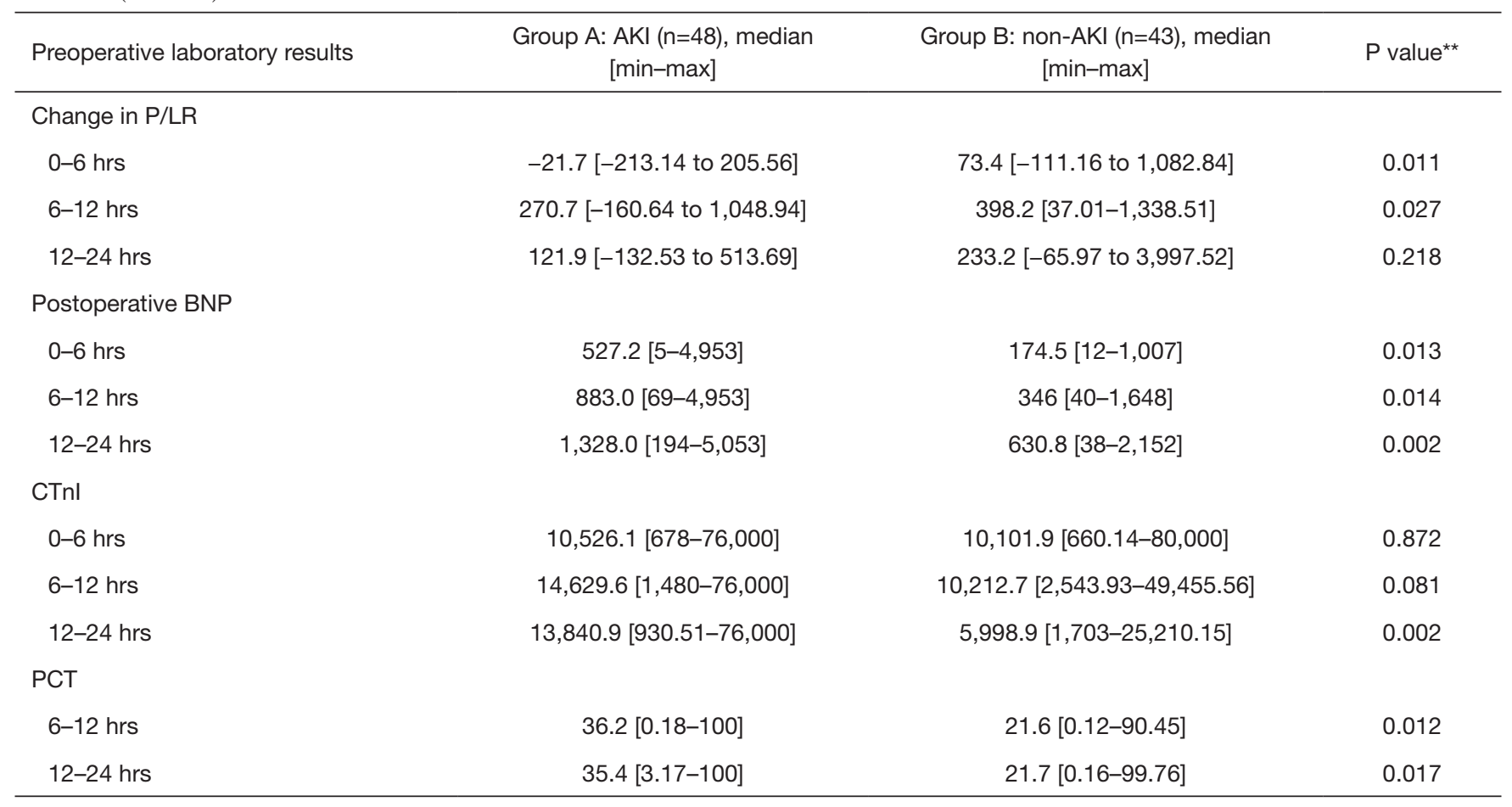

${ }^{\star \star}$, Student's $t$-test. AKI, acute kidney injury; CRP, C-reactive protein; N/LR, neutrophil-lymphocyte ratio; P/LR, platelet-lymphocyte ratio; BNP, brain natriuretic peptide; CTnl, troponin I; PCT, procalcitonin.

Table 4 Intraoperative data of the patients

\begin{tabular}{|c|c|c|c|}
\hline Characteristics & $\begin{array}{c}\text { Group A: AKI ( } n=48), \text { median [min- } \\
\max ]\end{array}$ & $\begin{array}{c}\text { Group B: non-AKI }(n=43), \text { median } \\
\text { [min-max] }\end{array}$ & $P$ value ${ }^{\star \star}$ \\
\hline Aortic cross clamp time (minutes) & $103.0[35-237]$ & $97.7[50-132]$ & 0.399 \\
\hline Duration of intraoperative hypotension (minutes) & $24.1[0-87]$ & $14.1[0-50]$ & 0.011 \\
\hline Total duration of hospital stay (days) & $26.2[0-213]$ & $15[0-37]$ & 0.018 \\
\hline
\end{tabular}

${ }^{\star \star}$, Student's $t$-test. AKI, acute kidney injury; CPB, cardiopulmonary bypass; ICU, intensive care unit.

groups. Significant differences were observed in the duration of hypotension during surgery $(\mathrm{P}=0.011)$ and length of hospital stay $(\mathrm{P}=0.018)$ between the groups.

Univariate and multivariate regression analyses of risk factors that were suggested to be influential in patients with AKI were conducted. The results are displayed in Table 5 . In the multivariate regression analysis, variables that were found to be statistically significant in the univariate analysis associated with postoperative AKI were analyzed. Through multivariate regression analysis, age $(\mathrm{P}=0.007)$, preoperative creatinine level $(\mathrm{P}=0.023)$, duration of intraoperative hypotension $(\mathrm{P}=0.027)$, and $\mathrm{P} / \mathrm{LR}(\mathrm{P}=0.043)$ at $6-12$ hours postoperatively were identified as independent predictors of CSA-AKI.

\section{Discussion}

In this retrospective study, an independent association was 
Table 5 Analysis of multiple risk factors of CSA-AKI during the perioperative period

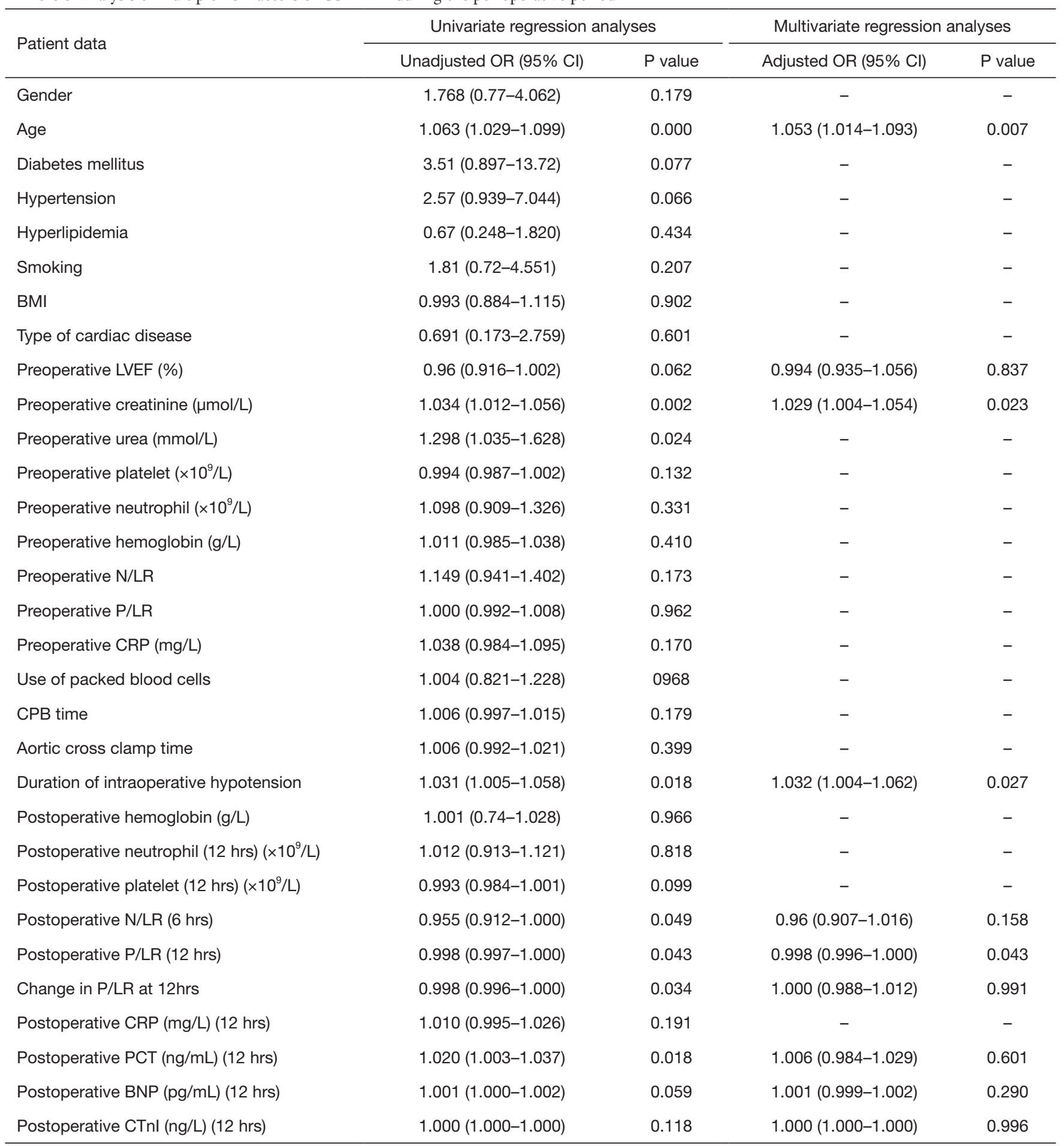

CSA-AKI, cardiac surgery-associated acute kidney injury; OR, odds ratio; Cl, confidence interval; BMI, body mass index; LVEF, left ventricular ejection fraction; N/LR, neutrophil-lymphocyte ratio; P/LR, platelet-lymphocyte ratio; CRP, C-reactive protein; PCT, procalcitonin; BNP, brain natriuretic peptide; CTnl, troponin I. 
observed between decreased P/LR and early postoperative AKI following cardiac surgery with $\mathrm{CPB}$ support. The pathophysiology of CSA-AKI is complex and is still not fully understood.

In terms of pathophysiology, the development of AKI following cardiac surgery may involve several major pathways, including hypoperfusion, ischemia-reperfusion injury, oxidative stress, inflammation, and neuro-humoral activation (7). Firstly, patients underwent cardiac surgery are often susceptible to renal hypoperfusion secondary to low-flow and non-pulsatile perfusion with fluctuation in body temperature during CPB. However, ischemiareperfusion may occur after $\mathrm{CPB}$ with the improvement in renal blood flow. Secondary, exposure of blood to CPB circuit triggers systemic inflammatory response, leading to surge of inflammatory cytokines and activation of completement pathways, then potentially subsequent AKI following cardiac surgery. Thirdly, the renin angiotensin aldosterone system and sympathetic nervous system could be activated by the hypoperfusion during cardiac surgery, possibly resulting in renal vasoconstriction and impaired renal perfusion (7).

The role of $\mathrm{P} / \mathrm{LR}$, which is a marker of chronic inflammation, has been studied in different cardiovascular conditions. As per well demonstrated, inflammation in the vessel wall plays an important role in the development atherosclerosis, potentially in process of coronary artery disease (11). Platelets release various proinflammatory factors that are important in all through the process of coronary artery disease and lymphocyte, as a marker of physical stress, is inversely related to inflammation. Ina recent systemic review, the increased P/LR, as a robust marker of inflammation, was reported with significant correlation with in-hospital mortality in patients with acute coronary syndrome, stable coronary artery disease and even heart failure (11). The correlation between AKI and decreased P/LR has rarely been reported. According to previous studies, decreased P/LR could potentially be attributed to a systemic inflammatory response provoked by $\mathrm{CPB}$ and micro-thrombotic events during $\mathrm{CPB}$ via the following mechanisms.

Firstly, platelets are regarded as a potent trigger in the pathogenesis of inflammatory activation in response to endothelial activation and neutrophil recruitment. Some evidence supports the critical role of platelets in the inflammatory response process, in which platelets interact with neutrophils, monocytes, and lymphocytes to modulate innate and adaptive immune responses $(12,13)$.
Also, platelets adhere to impaired, damaged endothelium and recruit leukocytes to amplify the inflammatory response (12).

Secondly, the connection between decreased P/ LR and CSA-AKI may be attributed to compromised microvascular circulation in the kidneys, which is related to the reduction in blood flow secondary to microthrombus. Platelets play a central role in coagulation and hemostasis in cases of surgical event or trauma. Postoperative thrombocytopenia might result from microvascular slugging and platelet consumption. Surgical trauma, ischemia-reperfusion, and $\mathrm{CPB}$ itself are potent triggers of the inflammatory response and have been found to facilitate the formation of micro-emboli consisting of activated leukocytes, fibrin, and aggregated platelets. As a result, this ischemic insult on renal endothelial cells facilitates the adhesion of platelets and leukocytes, amplifying inflammatory renal impairment. A previous retrospective trial reported a significant correlation between the lowest level of platelet counts and AKI following on-pump coronary artery bypass grafting (CABG) (8). During $\mathrm{CPB}$, platelets are activated via intrinsic pathways by contacting the circuit system, resulting in microvascular plugging with leukocytes and fibrin, and eventually renal ischemia and AKI (14). Therefore, thrombocytopenia resulting from $\mathrm{CPB}$ and decreased P/LR may demonstrate a pivotal role in the pathogenesis of CSA-AKI.

Given the depiction of decreased platelet counts in the process of CSA-AKI, postoperative thrombocytopenia might result from this microvascular sludging and platelet consumption. In this study, P/LR was employed as a marker of systemic inflammatory response and CSA-AKI. Compared to platelet count and lymphocyte alone, P/LR might be a superior marker to represent hypercoagulation and a hyperactive inflammatory response in some diseases (15-17). Although a previous study reported that platelet activation could be prevented by administration of antiplatelets and reduction in mortality with CSA-AKI (18), the administration of aspirin was limited due to concerns about perioperative hemorrhage.

Based on the fact that there is a limited pharmacological intervention that is effective for current AKI, it would be meaningful to predict postoperative AKI in the early stage. In this study, hematological parameters of the early postoperative period were reviewed according to the time sequence of the tests, so that the P/LR, N/LR, and platelet count of different time points can be compared accordingly. The hematological cell counts changed dramatically after surgery. As described previously, CPB is technically related 
to a decreased platelet count as a result of activation of both neutrophils and platelets, while surgical stress could lead to a suppression of cellular immunity. However, our data showed that there was no statistically significant difference in N/LR at these four different time points between the AKI and non-AKI groups. There was no correlation between postoperative AKI and perioperative N/LR, which represented the inflammatory status. These findings were inconsistent with previous studies $(15,19,20)$. There was a statistically significant difference in P/LR at 12 hours postoperatively, implying that P/LR in the early postoperative stage may potentially work as an indicator of AKI. According to our study, a decrease in P/LR by one measurement might indicate an increase in the risk of AKI by $2 \%$. However, this study failed to demonstrate a cutoff for P/LR to indicate the occurrence of AKI with a suboptimal area under the ROC curve (AUROC).

In addition to P/LR, our study also revealed advanced age, elevated preoperative serum creatinine level, and duration of intraoperative hypotension could potentially be predictive of postoperative AKI. It was previously well established that multiple risk factors throughout preoperative, intraoperative and postoperative periods are associated with occurrence of CSA-AKI, including advanced age, female gender, preoperative low left-ventricular ejection fraction, diabetes mellitus, high serum creatinine level, chronic obstructive pulmonary disease, prolonged CPB time, prolonged non-pulsatile perfusion during surgery and postoperative low cardiac output (21). In particular, direct factors related to occurrence of CSA-AKI might include exposure to nephrotoxic agents and suboptimal volume status of patients (5). Contrast agent is considered potentially nephrotoxic. However, it is not uncommon for coronary catheterization with contrast agent prior to cardiac surgery. International consensus suggested avoidance of contrast agents 24 to 72 hours prior to cardiac surgery, so that adequate recovery of renal cells could be optimized before subsequent surgical insult (21). Inappropriate perioperative volume status might closely relate to occurrence of CSA-AKI. Low cardiac output and overstrict fluid restriction could lead to renal hypoperfusion, activating renin-angiotensin-aldosterone system. Corresponding renal vasoconstriction accelerates the occurrence of CSA-AKI (5). Therefore, optimization of volume status plays an important role in prevention of CSA-AKI. Other prophylactic strategies of CSA-AKI might include the use of diuretics, mannitol and fenoldopam, which increases renal blood flow (7). Therapeutic management of AKI following cardiac surgery depends on the severity. Diuretics are commonly used to reduce fluid overload in patients with non-significant AKI while renal replacement therapy should be considered for presence of fatal fluid overload, electrolyte and acid-base disturbance (7).

Apart from P/LR, some emerging parameters are found to detect early postoperative AKI following cardiac surgery. Neutrophil gelatinase-associated lipocalin (NGAL), a highly induced protein in kidney secondary to ischemic or nephrotoxic insult, was considered as a sensitive and specific marker for early diagnosis of CSA-AKI. NGAL level in urine was found to increase significantly within 2 hours and decrease 6 hours following cardiac surgery in absence of rise in serum creatinine (5). Another promising biomarker is interleukin-18, which was reported to rise within 6 hours following cardiac surgery (5). Due to requirement of device, these markers are not widely utilized in clinical settings at this stage.

The authors acknowledge that the present study has some limitations that should be noted. Firstly, the data was collected in a single institution, which only functions as a regional hospital of the suburb, which is unfavorable for the generalizability of the results. Furthermore, the sample was from surgeries performed during the first 2 years, when protocols of cardiac surgeries were reviewed and optimized quarterly. This could be a potential bias affecting our results. Secondly, the source of bias should be considered when interpreting our results. As this is a retrospective study, the baseline laboratory values might not match between groups perfectly, which might impact on the development of AKI. Therefore, a large-scaled prospective study would be recommended for verifying the conclusion in the future.

\section{Conclusions}

In conclusion, this study demonstrated a correlation between decreased P/LR in the early postoperative stage and CSA-AKI. P/LR, as a surrogate of an inflammatory response, could be valuable as a marker for the timely prevention of AKI. If large-scaled prospective studies are approved, we should aim to demonstrate the casual relationship between P/LR and CSA-AKI.

\section{Acknowledgments}

Funding: None.

\section{Footnote}

Reporting Checklist: The authors have completed the 
STROBE reporting checklist. Available at https://dx.doi. org/10.21037/atm-21-6012

Data Sharing Statement: Available at https://dx.doi. org/10.21037/atm-21-6012

Conflicts of Interest: Both authors have completed the ICMJE uniform disclosure form (available at https://dx.doi. org/10.21037/atm-21-6012). The authors have no conflicts of interest to declare.

Ethical Statement: The authors are accountable for all aspects of the work in ensuring that questions related to the accuracy or integrity of any part of the work are appropriately investigated and resolved. According to local policy, informed consent was waived due to the retrospective nature of the study. All the procedures performed in this study were in accordance with the Declaration of Helsinki (as revised in 2013). The study was approved by institutional review board of School of Medicine, South China University of Technology.

Open Access Statement: This is an Open Access article distributed in accordance with the Creative Commons Attribution-NonCommercial-NoDerivs 4.0 International License (CC BY-NC-ND 4.0), which permits the noncommercial replication and distribution of the article with the strict proviso that no changes or edits are made and the original work is properly cited (including links to both the formal publication through the relevant DOI and the license). See: https://creativecommons.org/licenses/by-nc-nd/4.0/.

\section{References}

1. O'Neal JB, Shaw AD, Billings FT 4th. Acute kidney injury following cardiac surgery: current understanding and future directions. Crit Care 2016;20:187.

2. Wu B, Chen J, Yang Y. Biomarkers of Acute Kidney Injury after Cardiac Surgery: A Narrative Review. Biomed Res Int 2019;2019:7298635.

3. Gude D, Jha R. Acute kidney injury following cardiac surgery. Ann Card Anaesth 2012;15:279-86.

4. Suen WS, Mok CK, Chiu SW, et al. Risk factors for development of acute renal failure (ARF) requiring dialysis in patients undergoing cardiac surgery. Angiology 1998;49:789-800.

5. Mao H, Katz N, Ariyanon W, et al. Cardiac surgeryassociated acute kidney injury. Blood Purif 2014;37 Suppl
2:34-50.

6. Oshita T, Hiraoka A, Nakajima K, et al. A Better Predictor of Acute Kidney Injury After Cardiac Surgery: The Largest Area Under the Curve Below the Oxygen Delivery Threshold During Cardiopulmonary Bypass. J Am Heart Assoc 2020;9:e015566.

7. Wang Y, Bellomo R. Cardiac surgery-associated acute kidney injury: risk factors, pathophysiology and treatment. Nat Rev Nephrol 2017;13:697-711.

8. Kertai MD, Zhou S, Karhausen JA, et al. Platelet Counts, Acute Kidney Injury, and Mortality after Coronary Artery Bypass Grafting Surgery. Anesthesiology 2016;124:339-52.

9. Bu X, Zhang L, Chen P, et al. Relation of neutrophilto-lymphocyte ratio to acute kidney injury in patients with sepsis and septic shock: A retrospective study. Int Immunopharmacol 2019;70:372-7.

10. Bi JB, Zhang J, Ren YF, et al. Neutrophil-to-lymphocyte ratio predicts acute kidney injury occurrence after gastrointestinal and hepatobiliary surgery. World J Gastrointest Surg 2020;12:326-35.

11. Kurtul A, Ornek E. Platelet to Lymphocyte Ratio in Cardiovascular Diseases: A Systematic Review. Angiology 2019;70:802-18.

12. Gameiro J, Fonseca JA, Dias JM, et al. Neutrophil, lymphocyte and platelet ratio as a predictor of postoperative acute kidney injury in major abdominal surgery. BMC Nephrol 2018;19:320.

13. Li Z, Yang F, Dunn S, et al. Platelets as immune mediators: their role in host defense responses and sepsis. Thromb Res 2011;127:184-8.

14. Feindt P, Litmathe J, Boeken U, et al. Anticoagulation during extracorporeal circulation under conditions of an ongoing systemic inflammatory response syndrome: effects of heparin. Perfusion 2005;20:11-5.

15. Oylumlu M, Yıldız A, Oylumlu M, et al. Platelet-tolymphocyte ratio is a predictor of in-hospital mortality patients with acute coronary syndrome. Anatol J Cardiol 2015;15:277-83.

16. Azab B, Shah N, Akerman M, et al. Value of platelet/ lymphocyte ratio as a predictor of all-cause mortality after non-ST-elevation myocardial infarction. J Thromb Thrombolysis 2012;34:326-34.

17. Sunbul M, Gerin F, Durmus E, et al. Neutrophil to lymphocyte and platelet to lymphocyte ratio in patients with dipper versus non-dipper hypertension. Clin Exp Hypertens 2014;36:217-21.

18. Mangano DT; Multicenter Study of Perioperative Ischemia Research Group. Aspirin and mortality from 
coronary bypass surgery. N Engl J Med 2002;347:1309-17.

19. Parlar H, Şaşkın H. Are Pre and Postoperative Platelet to Lymphocyte Ratio and Neutrophil to Lymphocyte Ratio Associated with Early Postoperative AKI Following CABG? Braz J Cardiovasc Surg 2018;33:233-41.

20. Rinder CS, Fontes M, Mathew JP, et al. Neutrophil $\mathrm{CD} 11 \mathrm{~b}$ upregulation during cardiopulmonary bypass is associated with postoperative renal injury. Ann Thorac Surg 2003;75:899-905.

21. Tinica G, Brinza C, Covic A, et al. Determinants of acute kidney injury after cardiac surgery: a systematic review. Rev Cardiovasc Med 2020;21:601-10.

(English Language Editor: A. Kassem)

Cite this article as: He W, Zhou Y. The platelet-lymphocyte ratio is a promising predictor of early postoperative acute kidney injury following cardiac surgery: a case-control study. Ann Transl Med 2021;9(24):1751. doi: 10.21037/atm-21-6012 